\title{
Construction and Commisioning of the Beam delivery, storage ring and g-2 detectors at FNAL
}

\author{
A. Gioiosa* \\ Universita' degli studi del Molise, Pesche, Italy \\ INFN, Sezione di Lecce, Lecce, Italy \\ E-mail: antonio.gioiosa@gmail.com
}

\section{A. Anastasi}

Dipartimento MIFT, Università di Messina, Messina, Italy

on behalf of the Muon g-2 collaboration

The muon anomaly $a_{\mu}=(g-2) / 2$ has been measured to 0.54 parts per million by E821 experiment at Brookhaven, and at present there is a 3 to 4 -standard deviations difference between the Standard Model prediction and the experimental value. A new muon $g$-2 experiment, E989, is starting at Fermilab with the aim to improve the experimental error by a factor of four to clarify this difference. Some details of the beam delivery, storage ring and new experimental detector system of the $g-2$ experiment will be presented.

EPS-HEP 2017, European Physical Society conference on High Energy Physics 5-12 July 2017

Venice, Italy

${ }^{*}$ Speaker. 


\section{Introduction}

The muon anomaly $a_{\mu}=(g-2) / 2$ is a low-energy observable, which can be both measured and computed to high precision [1,2]. It provides an important test of the Standard Model (SM) and it is a sensitive search for new physics [3]. The result obtained from the precision measurement of $a_{\mu}$ by the E821 experiment at BNL [4], showed a discrepancy between its experimental value and the SM prediction. The major contribution to the uncertainty in the theoretical calculation are mainly due to the strong interaction effects which cannot be computed perturbatively at low energies. The experimental error achieved by the E821 experiment is $\delta a_{\mu}^{\mathrm{EXP}}=6.3 \times 10^{-10}$ $(0.54 \mathrm{ppm})$ [5]. The new experiment E989 at Fermilab aims to improve a fourfold factor with respect to the E821 experiment, reducing the systematic errors with the implementation of a more refined detector system and electronic together with an improvement of a factor 20 in statistics thanks to the Fermilab accelerator complex.

\section{Principle of the Muon g-2 experiment}

A beam of highly polarized muons of $3.1 \mathrm{GeV}$ is injected into the $g$-2 storage ring where a strong (1.45T) magnetic fields both traps the $\mu$ 's and causes their spin to precess. Muons momentum turns at the cyclotron frequency $\omega_{c}=\frac{e B}{\gamma m}$ while the spin rotates due to the combination of Larmor and Thomas precession $\omega_{s}=\frac{g e B}{2 m}+(1-\gamma) \frac{e B}{\gamma m}$.

Working at the precise muon momentum $p_{\mu}=3.1 \mathrm{GeV}$ causes that the difference of these frequencies to be independent of $\gamma$ and proportional to the anomalous magnetic moment $\omega_{a}=\omega_{s}-\omega_{c}=$ $\frac{a_{\mu} e B}{m}$.

The quantity $a_{\mu}$, is obtained by measuring the frequency $\omega_{a}$ and the magnetic field $B$, which can be tied to the precession frequency of free protons $\omega_{p}$. The $\omega_{a}$ measurement is performed by detecting the high energy decay positrons emitted in the muon weak decay in the 24 calorimeter stations placed around the storage ring. This is made possible thanks to the parity-violating nature of the weak decay of the muon which leads to a strong correlation between the muon spin and the high energy positron emitted. At the same time precise knowledge of the magnetic field is obtained by Nuclear Magnetic Resonance (NMR) probes that relates the proton precession frequency $\omega_{p}$ to the absolute field experienced by muons.

\section{Ring and beam}

The 14 meters BNL storage ring of the E821 experiment was relocated to Fermilab in 2013. It permits to store muons in a high uniform magnetic field. Critical issue for the experiment was the reinstallation of the storage ring. This procedure started immediately once the new MC-1 building was ready. The magnet is fully operational and shimmed at $\pm 25 \mathrm{ppm}$ and all the detectors are installed and operational. Another important point are the upgrades of the Fermilab accelerator complex. One of this upgrades is related to the conversion of the antiproton complex to a muon source capable to delivery a high-purity $3.1 \mathrm{GeV}$ muon beam to the Muon $g-2$ experiment. Commissioning started in 2017 and the first beam arrived in June. 


\section{Detectors}

\subsection{Calorimeter}

The new calorimeters of the E989 consist of 24 stations placed around the ring. Each calorimeter is an $9 \times 6$ array of pure $\mathrm{PbF}_{2}$ Cerenkov crystals readout by SiPM. The higly segmentation allow for a better spatial resolution of pileup events while the fast nature of Cerenkov radation improves time resolution [6]. SiPMs are suitable for the application inside high magnetic field with no interference and a stable voltage distribution is necessary to maintain gain stability together with a dedicated Laser Calibration System, in order to improve the systematic errors due to gain fluctuations. All the calorimeters are installed around the ring and succesfully tested during the June test run $^{1}$, see Fig.1.

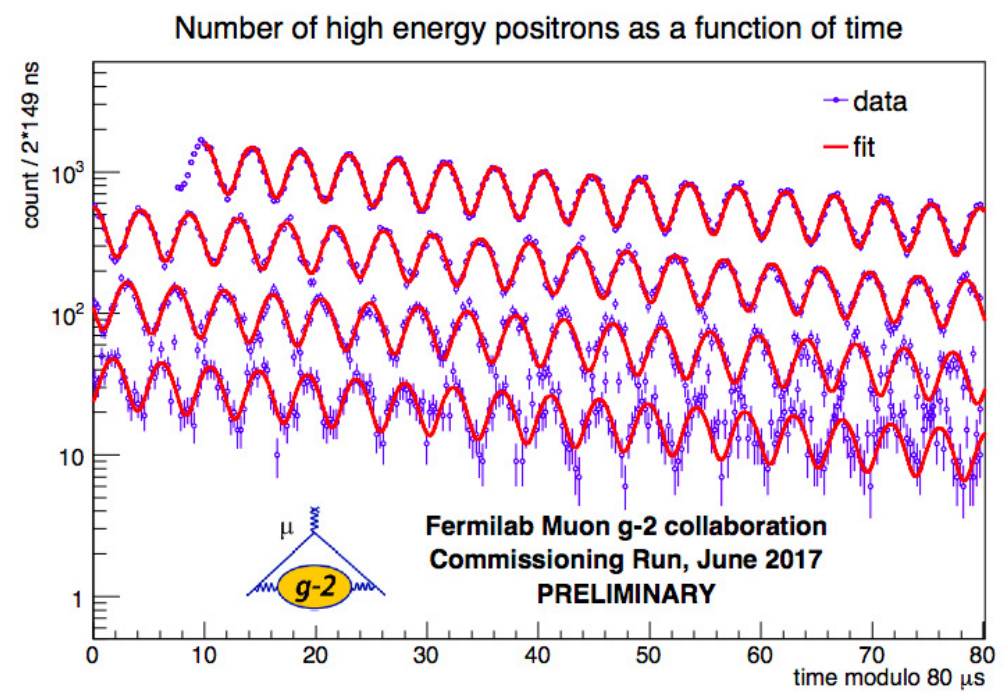

Figure 1: The figure was accumulated from two weeks of data accumulated in June 2017 and has approximately 700k positrons. The number of wiggles is somewhere between that achieved by CERN-II and CERN-III.

\subsection{Tracking system}

A tracking system has been developed to reduce systematic error due to beam dynamics inside the storage ring. Three detectors will be installed along the ring. Each detector is composed by 8 modules of mylar straw fibers and dedicated electronic [7].

Laboratory test confirmed high level efficiency of $99.2 \%$ for a single module.

All the 8 modules of the first tracker detector have been installed and commissioned during the test run.

\subsection{Laser Calibration System}

A high performance calibration system is required for the on-line monitoring of the output stability of each individual tower in all calorimeter stations. It is estimated that the detector re-

\footnotetext{
${ }^{1}$ The test run took place from June 1 to July 7.
} 
sponse must be calibrated with relative accuracy at sub-per mil level to achieve the goal of the E989 experiment to keep systematics contributions to the accuracy on $\omega_{a}$ at $0.02 p p m$ level. This is a challenge for the design of the calibration system because the desired accuracy is at least one order of magnitude higher than that of all other existing, or adopted in the past, calibration systems for calorimetry in particle physics.

As almost 1300 channels must be kept calibrated during data taking, the proposed solution is based on the method of sending simultaneous light calibration pulses onto the readout photodetector through the crystals of the calorimeter. Light pulses should be stable in intensity and timing in order to correct for systematic effects due to drifts in the response of the crystal readout devices. A suitable photo-detector system must be included in the calibration architecture to monitor any fluctuation of the light. The guidelines given by the experiment to define in the correct way the architecture of the entire system can be found in [8]. Several test beam were performed to test all the performance of the system. The final design of the Laser Calibration System installed at Fermilab is shown in Fig.2. The system has been heavily used during the test run.

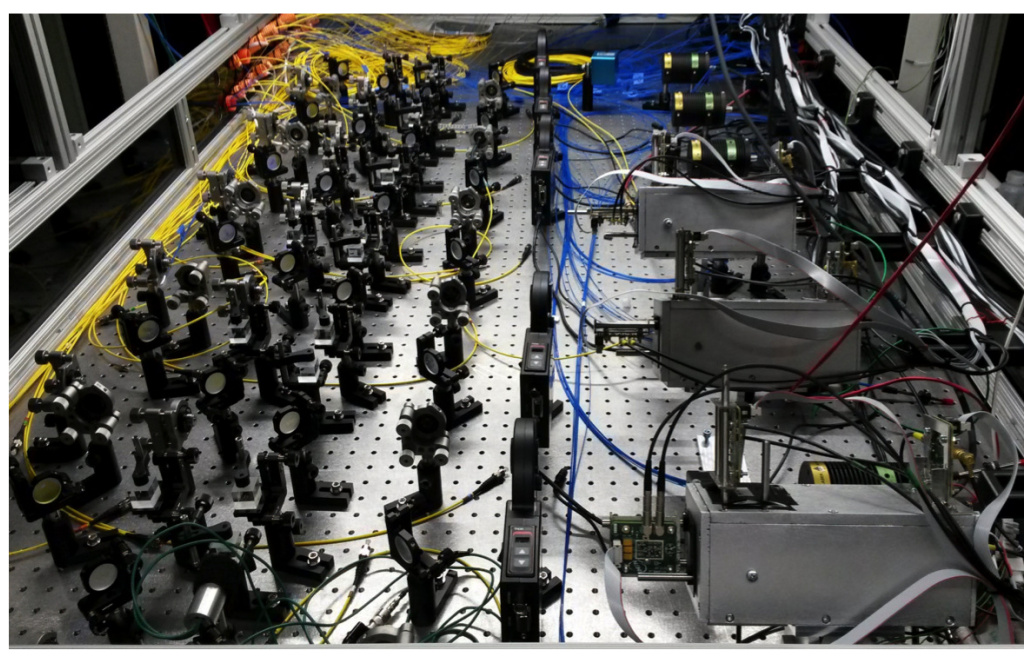

Figure 2: The Laser Calibration System optica table.

\section{Conclusion}

A five weeks commissioning run has been completed successfully in July and the first commissioning data analysis has started. The next run is scheduled for mid of October 2017. The goal is to reach the BNL level precision with the data taken in early 2018, and the final $0.14 \mathrm{ppm}$ result measurement in 2020 . This will require a total of $1.5 \times 10^{11}$ collected events.

\section{References}

[1] F. Jegerlehner, "The anomalous magnetic moment of the muon," Springer Tracts Mod. Phys. 226 (2008) 1 
[2] T. Blum, A. Denig, I. Logashenko, E. de Rafael, B. Lee Roberts, T. Teubner and G. Venanzoni, arXiv:1311.2198 [hep-ph]

[3] D. Stöckinger, "Muon (g-2) and physics beyond the standard model," In Roberts, Lee B., Marciano, William J. (eds.): Lepton dipole moments 393-438 (Advanced series on directions in high energy physics. 20)

[4] H. N. Brown et al. [Muon g-2 Collaboration], Phys. Rev. Lett. 86 (2001) 2227

[5] G. W. Bennett et al. [Muon g-2 Collaboration], "Final Report of the Muon E821 Anomalous Magnetic Moment Measurement at BNL” Phys. Rev. D. 73 (2006) 072003

[6] New Muon (g-2) Collaboration, R.M. Carey et. al., see http://lss.fnal.gov/archive/testproposal/0000/fermilab-proposal0989.shtml A. T. Fienberg et al., Studies of an array of PbF 2 Cherenkov crystals with large area SiPM read-out, Nucl. Instrum. Meth A783 12-21 (2015).

[7] M. Rominsky, B. Casey, L. Welty-Rieger, H. Schellman, M. Eads and N. Pohlman, “T-1042: Muon g-2 Straw Tracker," FERMILAB-PROPOSAL-1042.

[8] A. Anastasi et al., Test of candidate light distributors for the muon ( $g$-2) experiment, Nucl. Instrum. Meth. A788 43-48 (2015).

[9] A. Anastasi et al., Electron beam test of key elements of the laser-based calibration system for the muon g-2 experiment, sub. Nucl. Instrum Meth. A (2016). 